\title{
Effect of Adenylyl Cyclase Type 6 on Localized Production of cAMP by $\beta-2$ Adrenoceptors in Human Airway Smooth-Muscle Cells ${ }^{[}$
}

\author{
Shailesh R. Agarwal, Chase Fiore, Kathryn Miyashiro, Rennolds S. Ostrom, \\ and Robert D. Harvey \\ Department of Pharmacology, University of Nevada, Reno School of Medicine, Reno, Nevada (S.R.A., C.F., K.M., R.D.H.); and \\ Department of Biomedical and Pharmaceutical Sciences, Chapman University School of Pharmacy, Irvine, California (R.S.O.)
}

Received January 18, 2019; accepted May 2, 2019

\begin{abstract}
$\beta_{2}$-Adrenoceptors ( $\beta_{2} \mathrm{ARs}$ ) are concentrated in caveolar lipid raft domains of the plasma membrane in airway smooth-muscle (ASM) cells, along with adenylyl cyclase type 6 (AC6). This is believed to contribute to how these receptors can selectively regulate certain types of cAMP-dependent responses in these cells. The goal of the present study was to test the hypothesis that $\beta_{2} A R$ production of cAMP is localized to specific subcellular compartments using fluorescence resonance energy transfer-based cAMP biosensors targeted to different microdomains in human ASM cells. Epac2-MyrPalm and Epac2-CAAX biosensors were used to measure responses associated with lipid raft and nonraft regions of the plasma membrane, respectively. Activation of $\beta_{2} A R s$ with isoproterenol produced cAMP responses that are most readily detected in lipid raft domains. Furthermore, overexpression of AC6 somewhat
\end{abstract}

paradoxically inhibited $\beta_{2} A R$ production of cAMP in lipid raft domains without affecting $\beta_{2} A R$ responses detected in other subcellular locations or cAMP responses to $\mathrm{EP}_{2}$ prostaglandin receptor activation, which were confined primarily to nonraft domains of the plasma membrane. The inhibitory effect of overexpressing AC6 was blocked by inhibition of phosphodiesterase type 4 (PDE4) activity with rolipram, inhibition of protein kinase $A(P K A)$ activity with $\mathrm{H} 89$, and inhibition of $A$ kinase anchoring protein (AKAP) interactions with the peptide inhibitor $\mathrm{Ht} 31$. These results support the idea that overexpression of AC6 leads to enhanced feedback activation of PDE4 via phosphorylation by PKA that is part of an AKAP-dependent signaling complex. This provides insight into the molecular basis for localized regulation of cAMP signaling in human ASM cells.

\section{Introduction}

$\beta_{2}$-Adrenoceptor $\left(\beta_{2} \mathrm{AR}\right)$ agonists are a mainstay of therapy for asthma, chronic obstructive pulmonary disease, and other respiratory disorders. $\beta_{2} \mathrm{AR}$ activation induces airway smooth muscle (ASM) relaxation and bronchodilation by stimulating adenylyl cyclase (AC) activity and the production of cAMP. Although cAMP plays a central role in regulating the contractile state of ASM (Pelaia et al., 2008; Noble et al., 2014), this ubiquitous signaling molecule is also involved in eliciting a number of other responses in the muscle cells that make up this tissue (Billington et al., 2013). The specific effect mediated by cAMP often depends on the signaling pathway responsible for its production. In ASM cells, cAMP production is regulated

This work was supported by the National Institutes of Health [Grants R01 GM107094, R01 GM101928, and R01 HL145778]. S.R.A. was supported by a National Institute of General Medical Sciences Center of Biological Research Excellence [P20 GM130459].

https://doi.org/10.1124/jpet.119.256594.

S This article has supplemental material available at jpet.aspetjournals.org. by both $\beta_{2} \mathrm{ARs}$ and $\mathrm{EP}_{2}$ prostaglandin receptors $\left(\mathrm{EP}_{2} \mathrm{Rs}\right)$ (Bogard et al., 2011). Production of cAMP by either type of receptor is capable of causing human ASM relaxation; however, only cAMP produced by $\beta_{2} \mathrm{ARs}$ stimulates cytoskeletal reorganization and cell-shape changes associated with arborization, and only cAMP produced by $\mathrm{EP}_{2} \mathrm{Rs}$ increases the expression of the cytokine interleukin-6 (Bogard et al., 2012, 2014). Understanding how cells compartmentalize cAMP and generate unique responses upon activation of a given receptor is important because it may help explain why $\beta_{2} \mathrm{AR}$ agonists, which are widely used as bronchodilators, suffer from issues with tachyphylaxis owing to receptor desensitization (Penn et al., 1998) and increased adverse effects (Taylor, 2009).

The simplest way to explain the ability of different receptors to illicit unique functional responses is if each receptor stimulates cAMP production that is confined to distinct subcellular locations. For this to be true, however, these receptors must then be distributed in distinctly different physical locations. Consistent with this hypothesis, $\beta_{2} \mathrm{ARs}$ and $\mathrm{EP}_{2} \mathrm{Rs}$ have been found to reside in different fractions of the plasma

ABBREVIATIONS: AC6, adenylyl cyclase type 6; AKAP, A kinase-anchoring protein; ASM, airway smooth muscle; $\beta_{2}$ AR, $\beta_{2}$-adrenoceptor; ECFP, enhanced cyan fluorescent protein; $\mathrm{EP}_{2} \mathrm{R}, \mathrm{EP}_{2}$ prostaglandin receptor; Epac2-CAAX, Epac2-camps biosensor with a prenylation targeting sequence; Epac2-camps, exchange protein activated by cAMP type 2-based cAMP biosensor; Epac2-MyrPalm, Epac2-camps biosensor with an acylation targeting sequence; EYFP, enhanced yellow fluorescent protein; FRET, fluorescence resonance energy transfer; IBMX, 3-isobutyl-1methylxanthine; Iso, isoproterenol; PDE4, phosphodiesterase type 4; PKA, protein kinase A. 
membrane (Ostrom et al., 2001; Bogard et al., 2011, 2012). Most cell membranes contain cholesterol- and sphingomyelinrich domains referred to as lipid rafts, which form buoyant membrane fractions on a sucrose density gradient (Insel et al., 2005; Ostrom and Insel, 2006). Caveolae are represented by a subset of the buoyant fractions associated specifically with caveolins, which are scaffolding proteins involved in forming signaling complexes. In human ASM cells, $\beta_{2} \mathrm{ARs}$ are found specifically in caveolar, lipid raft fractions of the plasma membrane, whereas $\mathrm{EP}_{2} \mathrm{Rs}$ are found in the nonraft membrane fractions (Bogard et al., 2011).

$\mathrm{ASM}$ responses to $\beta_{2} \mathrm{AR}$ and $\mathrm{EP}_{2} \mathrm{R}$ stimulation have also been attributed to activation of different isoforms of adenylyl cyclase (AC), the enzyme responsible for cAMP production. AC type 6 (AC6) is found specifically in caveolar membrane fractions of ASM cells, and its overexpression selectively enhances $\beta_{2} \mathrm{AR}$ production of cAMP and cAMP-dependent arborization. AC type 2 (AC2) is found exclusively in nonraft fractions of the plasma membrane, and its overexpression selectively enhances $\mathrm{EP}_{2} \mathrm{R}$ stimulation of cAMP and cAMP-dependent production of interleukin-6 (Bogard et al., 2011, 2012, 2014).

The purpose of the present study was to test the hypothesis that in primary human ASM cells, $\beta_{2} \mathrm{AR}$ stimulation preferentially enhances cAMP production in subcellular locations associated with lipid raft domains of the plasma membrane by a mechanism involving activation of AC6. This was done by comparing cAMP responses detected by Epac2-camps, a fluorescence resonance energy transfer (FRET)-based biosensor, expressed in different subcellular locations (Nikolaev et al., 2004). Epac2-camps itself has no targeting sequences and is expressed throughout the cytoplasmic domain. Epac2-MyrPalm is a version of the probe that contains an acylation sequence targeting it to lipid raft domains of the plasma membrane, and Epac2-CAAX contains a prenylation sequence that targets the probe to nonraft domains of the plasma membrane. These sequences have been previously shown to target proteins to lipid raft and nonraft fractions of the plasma membrane of various cell types (Melkonian et al., 1999; Zacharias et al., 2002; Depry et al., 2011). Furthermore, our previous work has also demonstrated that probes targeted using these sequences are capable of detecting differences in responses produced by receptors located in lipid raft and nonraft membrane domains of various cell types (Agarwal et al., 2014, 2017, 2018). In human ASM cells, we demonstrated the $\mathrm{EP}_{2} \mathrm{Rs}$ preferentially couple to AC2 to produce changes in cAMP that are confined primarily to nonraft domains of the plasma membrane (Agarwal et al., 2017). In the present study, we demonstrate that $\beta_{2} \mathrm{ARs}$ produce responses that are primarily associated with lipid raft domains. Furthermore, AC6 regulates this response through a mechanism that involves direct interaction with protein kinase A (PKA) and positive feedback activation of PDE4.

\section{Materials and Methods}

Cell Culture. Human ASM cells were derived from tracheae and primary bronchi from nonasthmatic human donors by Dr. Raymond

\section{Epac2-camps}
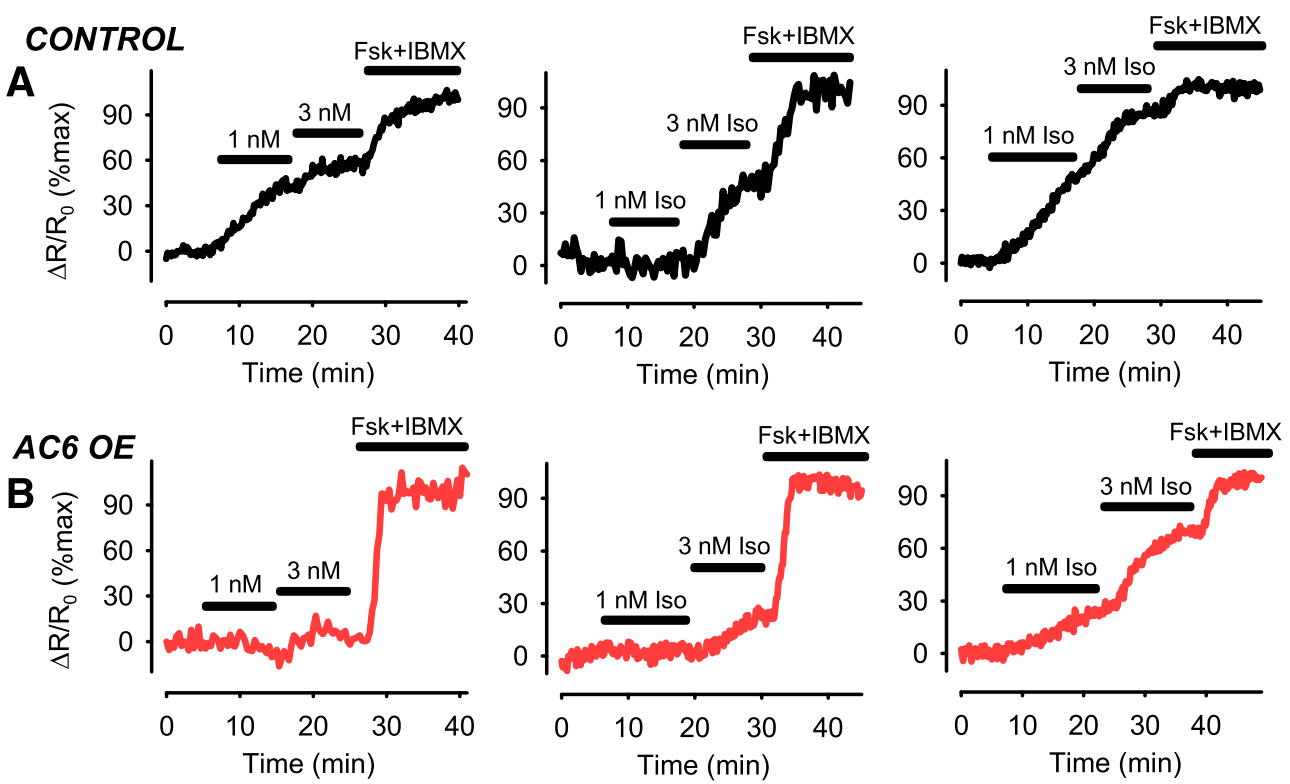

C
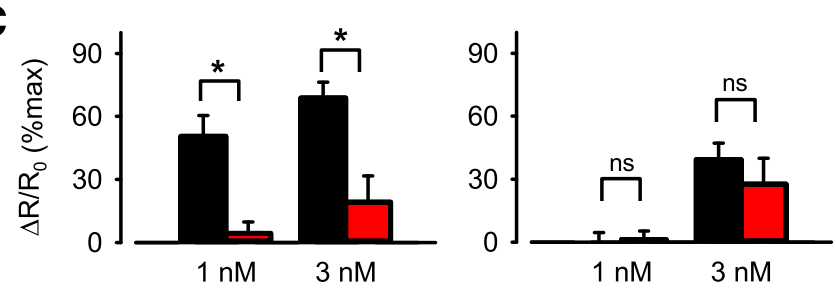

isoproterenol

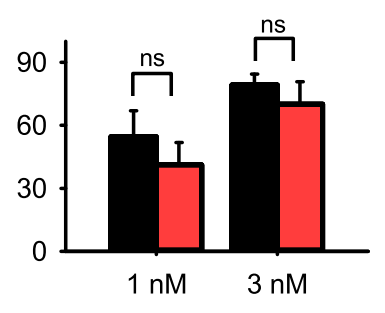

isoproterenol
Fig. 1. Changes in cAMP activity in different subcellular domains after $\beta_{2} \mathrm{AR}$ stimulation. Representative time course of changes in magnitude of FRET response $\left(\Delta \mathrm{R} / \mathrm{R}_{0}\right)$ detected by Epac2-MyrPalm, Epac2-CAAX, and Epac2-camps biosensors after activation of $\beta_{2} \mathrm{ARs}$ with 1 and $3 \mathrm{nM}$ isoproterenol (Iso) in (A) untreated human airway smooth-muscle (ASM) cells (control) and (B) human ASM cells overexpressing (OE) AC6. Results are normalized to the magnitude of the maximal response produced by forskolin (FSK, $10 \mu \mathrm{M})$ and IBMX $(100 \mu \mathrm{M})$ in the same cell. (C) Size of average FRET responses in control cells (black bars) and AC6 OE cells (red bars). AC6 OE caused a significant $(P<0.05$, student's $t$ test) decrease in the magnitude of the responses detected by Epac2-MyrPalm (control, $\mathrm{n} / N=6 / 6$; AC6 OE, $\mathrm{n} / N=9 / 4$ ), but not those detected by Epac2-CAAX (control, $\mathrm{n} / N=7 / 6$; AC6 $\mathrm{OE}, \mathrm{n} / N=6 / 6$ ), or Epac2-camps (control, $\mathrm{n} / N=6 / 3 ;$ AC6 OE, $\mathrm{n} / N=5 / 3$ ). 
Penn (Thomas Jefferson University, Philadelphia, PA) and Dr. Reynold Panettieri (Rutgers University, New Brunswick, NJ), as described previously (Yan et al., 2011). These cells come from deidentified biologic samples that are considered exempt by the University of Nevada, Reno, and Chapman University Institutional Review Boards. Experiments were conducted using multiple samples of primary cells derived from six different patients representing both sexes. All cells used were between passage 6 and 9. Cells were maintained in Ham's F12 nutrient mixture supplemented with $10 \%$ fetal bovine serum, $100 \mathrm{U} / \mathrm{ml}$ penicillin, $100 \mu \mathrm{g} / \mathrm{ml}$ streptomycin. Cells were transduced with adenovirus constructs containing Epac2based cAMP biosensors for 48-72 hours. For overexpression experiments, adenoviral constructs expressing AC6 and/or Ht31 were used at titers previously shown to produce $>90 \%$ transduction efficiency (Bogard et al., 2011). All experiments were conducted at room temperature.

FRET Microscopy. The Epac2-camps, Epac2-MyrPalm, and Epac2-CAAX biosensors used in the present study have been previously characterized (Nikolaev et al., 2004; Agarwal et al., 2014, 2017, 2018). All three probes have a similar affinity for activation by cAMP (Agarwal et al., 2014). Live-cell imaging experiments were conducted as described previously (Warrier et al., 2005; Warrier et al., 2007; Iancu et al., 2008; Agarwal et al., 2017). Briefly, cells were bathed in the following solution (in $\mathrm{mM}$ ): $\mathrm{NaCl} 137, \mathrm{KCl} 5.4, \mathrm{MgCl}_{2} 0.5, \mathrm{CaCl}_{2}$ 1.0, $\mathrm{NaH}_{2} \mathrm{PO}_{4}$ 0.33, glucose 5.5, and HEPES 5 (pH 7.4). FRET imaging was conducted using an Olympus IX71 inverted microscope (Olympus America, Center Valley, PA) equipped with a Hamamatsu OrcaD2 dual chip CCD camera and HCImage data acquisition and analysis software (Hamamatsu Corporation, Bridgewater, NJ). Images were obtained with a $40 \times$ water-immersion objective ( 1.3 numerical aperture). ECFP excitation was achieved by using a $300-\mathrm{W}$ xenon arc lamp with a D436/20 band-pass filter and a 455DCLP dichroic mirror. ECFP and EYFP (FRET) emissions were measured simultaneously using a 505DCXR dichroic mirror with D480/30 and D535/30 band-pass filters. Changes in cAMP activity were defined as the change in background and bleed-through corrected ECFP/EYFP fluorescence intensity ratio $(\Delta \mathrm{R})$ relative to the baseline ratio measured in a region of interest. As long as the nucleus was avoided, moving the ROI did not significantly affect the results. Ratiometric measurements also ensured that the responses measured were independent of the level of expression of the probes. FRET ratios were measured once every 10 seconds. A baseline of at least 5 minutes was established before application of any drug. Because the response to low concentrations of agonists were relatively slow to develop, all drugs were applied for at least 10 minutes before their effects were measured. Responses were calculated relative to the average ratio measured over the 30 -second period immediately preceding application of any drug. To control for any differences in the dynamic range of the FRET responses produced by the different probes, all results were normalized to the magnitude of the maximal probe response observed in the same cell after exposure to maximally stimulating concentrations 3-isobutlyl-1-methylxanthine (IBMX), a nonspecific PDE inhibitor, plus forskolin, a direct activator of AC activity.

Materials. Ham's F12 medium, penicillin, streptomycin, and fetal bovine serum were purchased from Life Technologies (Carlsbad, CA). Rolipram and forskolin were obtained from Tocris Bioscience (Bristol, UK). All other reagents were purchased from Sigma-Aldrich (St. Louis, MO).

Statistics. All data are expressed as the mean \pm S.E.M. of the indicated number of experiments conducted using different cells $(n)$ obtained from different preparations $(N)$. Statistical significance $(P<0.05)$ was determined by Student's $t$ test with Holm-Sidak correction, where appropriate, using SigmaPlot (Systat Software, San Jose, CA).
Epac2-MyrPalm

\section{CONTROL}

A
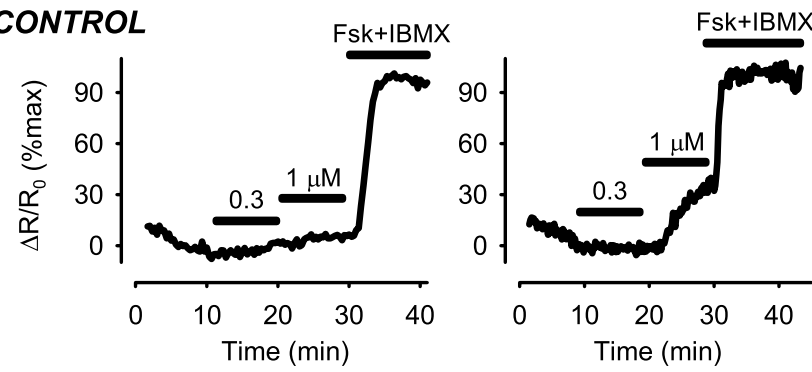

Epac2-CAAX

\section{AC6 OE}
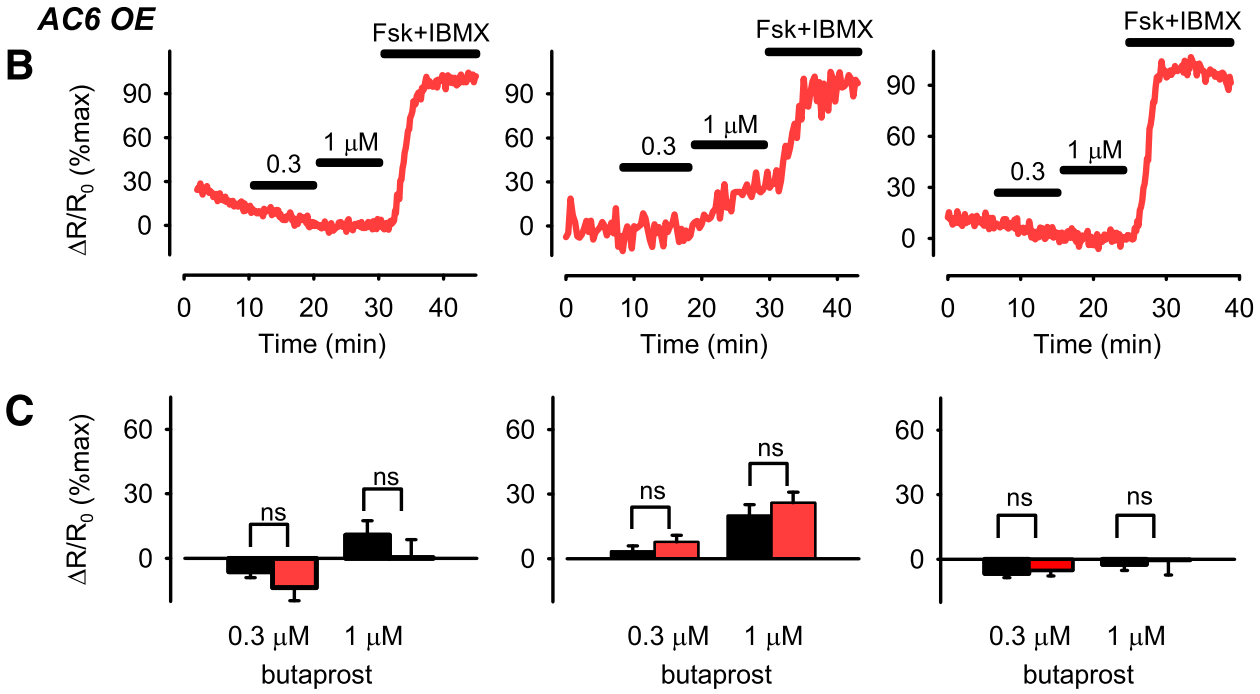

Fig. 2. Changes in cAMP activity in different subcellular domains after $\mathrm{EP}_{2} \mathrm{R}$ stimulation. Representative time course of changes in magnitude of FRET response $\left(\Delta \mathrm{R} / \mathrm{R}_{0}\right)$ detected by Epac2-MyrPalm, Epac2-CAAX, and Epac2-camps biosensors after activation of $\mathrm{EP}_{2} \mathrm{Rs}$ with 0.3 and $1 \mu \mathrm{M}$ butaprost in (A) untreated human ASM cells (control) and (B) human ASM cells overexpressing (OE) AC6. Results are normalized to the magnitude of the maximal response produced by forskolin (FSK, $10 \mu \mathrm{M})$ and IBMX $(100 \mu \mathrm{M})$ in the same cell. (C) Size of average FRET responses in control cells (black bars) and AC6 OE cells (red bars). AC6 OE did not significantly affect $(\boldsymbol{P}>0.05$, Student's $t$ test $)$ responses detected by Epac2-MyrPalm (control, $\mathrm{n} / N=13 / 8 ;$ AC6 OE, $\mathrm{n} / N=8 / 5)$, Epac2CAAX (control, $\mathrm{n} / N=20 / 10 ;$ AC6 OE, $\mathrm{n} / N=$ 10/7), or Epac2-camps (control, n/8 = 13; AC6 $\mathrm{OE}, \mathrm{n} / N=11 / 6)$ 


\section{Results}

Responses to $\beta_{2} \mathrm{AR}$ stimulation were measured using submaximally stimulating concentrations of the agonist isoproterenol (Iso). In control cells, exposure to $1 \mathrm{nM}$ Iso elicited responses that were detected by the cytosolic (Epac2-camps) and lipid raft (Epac2-MyrPalm) targeted probes, but not by the nonraft targeted (Epac2-CAAX) probe (Fig. 1A). Exposure to $3 \mathrm{nM}$ Iso elicited responses detected by all three probes, although the response detected by Epac2-CAAX was significantly smaller (Fig. 1C). These results are consistent with the idea that $\beta_{2} \mathrm{ARs}$ are concentrated primarily in lipid raft domains of the plasma membrane of human ASM cells.

We then examined the effect of overexpressing AC6, but this did not affect the responses to maximally stimulating concentrations of forskolin plus IBMX (see Supplemental Fig. 1); however, it did significantly reduce the relative magnitude of the response to $\beta_{2} \mathrm{AR}$ stimulation detected by Epac2-MyrPalm $(P<0.05)$ without altering the responses detected by Epac2camps or Epac2-CAAX $(P>0.05)$ (Fig. 1, B and C). This finding supports the idea that AC6 is targeted primarily to lipid raft domains of the plasma membrane, where it affects $\beta_{2} \mathrm{AR}$-mediated production of cAMP. Despite the fact that AC6 overexpression selectively altered $\beta_{2} \mathrm{AR}$ responses associated with lipid raft domains, our original hypothesis was that it would enhance the production of cAMP. Contrary to this prediction, AC6 overexpression actually inhibited the $\beta_{2} \mathrm{AR}$ response detected by Epac2-MyrPalm.

To determine whether the effect of overexpressing AC6 is specific to $\beta_{2} \mathrm{ARs}$, we also looked at the effect it had on responses to $\mathrm{EP}_{2}$ prostaglandin receptor $\left(\mathrm{EP}_{2} \mathrm{R}\right)$ activation using the agonist butaprost. As reported previously, in control cells, butaprost elicited cAMP responses that were most readily detected by the Epac2-CAAX probe in nonraft domains of the plasma membrane (Fig. 2A) (Agarwal et al., 2017). Furthermore, overexpressing AC6 neither enhanced nor inhibited cAMP responses to butaprost $(P>0.05)$ (Fig. 2, B and $C$ ) detected by any of the probes. This result further supports the idea that AC6 is selectively associated with regulating $\beta_{2} \mathrm{AR}$ responses in lipid raft domains of the plasma membrane.

We hypothesized that AC6 overexpression causes a localized decrease in cAMP produced by $\beta_{2} \mathrm{ARs}$ in lipid raft domains as a result of an increase in phosphodiesterase (PDE) activity through a positive feedback mechanism involving protein kinase A (PKA) (Conti and Beavo, 2007). The activity of PDE4, which is prominent in airway smoothmuscle cells (Méhats et al., 2003; Bogard et al., 2012), has been shown to be upregulated by PKA-dependent phosphorylation. To examine this possibility, we looked at the effect of rolipram $(10 \mu \mathrm{M})$, a selective PDE4 inhibitor, on $\beta_{2} \mathrm{AR}$ responses in control cells, as well as cells overexpressing AC6 (Fig. 3). Rolipram by itself had no effect on cAMP activity detected by the Epac2-MyrPalm probe in either control or AC6-overexpressing cells $(P>0.05)$. Furthermore, rolipram did not appear to alter the magnitude of the response to Iso in control cells (Fig. 3A); however, it did significantly alter the response to Iso in AC6- overexpressing cells (Fig. 3, B and $\mathrm{C}$ ). In the presence of rolipram, the response to Iso was not significantly different from control cells $(P>0.05)$, in contrast to the significant decrease in magnitude of the $\beta_{2} \mathrm{AR}$ response associated with AC6 overexpression in the absence
Epac2-MyrPalm
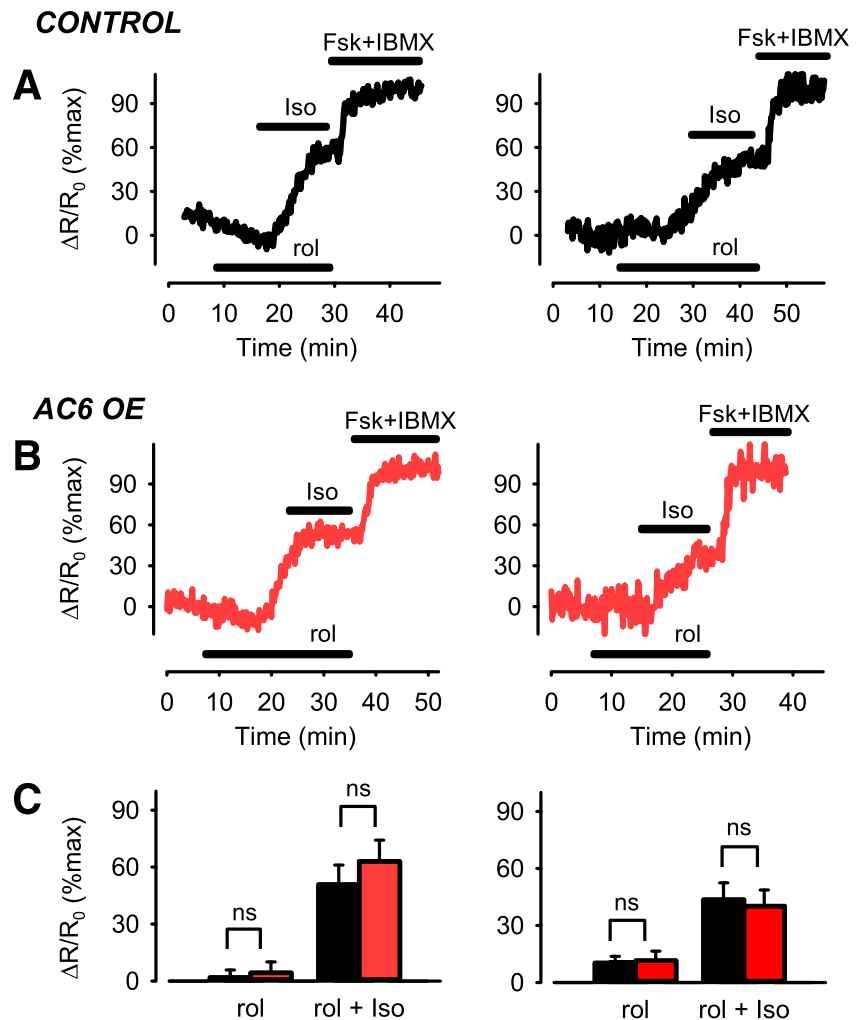

Fig. 3. Changes in cAMP activity associated with lipid raft and nonraft plasma membrane domains after $\beta_{2} \mathrm{AR}$ stimulation in the presence of PDE4 inhibition. Representative time course of changes in magnitude of FRET response $\left(\Delta \mathrm{R} / \mathrm{R}_{0}\right)$ detected by Epac2-MyrPalm and Epac2-CAAX biosensors after inhibition of PDE4 activity with rolipram $(10 \mu \mathrm{M})$ and subsequent activation of $\beta_{2} \mathrm{ARs}$ with $3 \mathrm{nM}$ isoproterenol (Iso) in (A) untreated human ASM cells (control) and (B) human ASM cells overexpressing (OE) AC6. Results are normalized to the magnitude of the maximal response produced by forskolin (FSK, $10 \mu \mathrm{M}$ ) and IBMX $(100 \mu \mathrm{M})$ in the same cell. (C) Size of average FRET responses in control cells (black bars) and AC6 OE cells (red bars). In the presence of rolipram, there were no significant ( $P>0.05$, Student's $t$ test) differences in the magnitude of the responses to Iso detected by Epac2-MyrPalm in control $(\mathrm{n} / N=6 / 5)$ and AC6 OE $(\mathrm{n} / N=6 / 4)$ cells or those detected by Epac2-CAAX in control $(\mathrm{n} / N=9 / 5)$ and AC6 OE $(\mathrm{n} / N=9 / 6)$ cells.

of PDE4 inhibition (see Fig. 1). Furthermore, this effect appeared to be specific for responses associated with lipid raft domains, as rolipram did not enhance the response to Iso detected by Epac2-CAAX in nonraft domains of AC6overexpressing cells (Fig. 3, B and C); it was the same size as the response to Iso in control cells in the absence of rolipram (see Fig. 1, A and C). This result supports the idea that AC6 overexpression leads to a localized decrease in cAMP produced by $\beta_{2} \mathrm{ARs}$ associated specifically with lipid raft domains that is mediated by PDE4.

The preceding results are consistent with the hypothesis that overexpressing AC6 leads to an increase in cAMP production, which in turn acts in a positive feedback manner to enhance PDE4 activity, presumably through a mechanism involving PKA-dependent phosphorylation (Conti and Beavo, 2007). To examine this possibility, we compared the response to Iso in cells first exposed to the PKA inhibitor H89 $(1 \mu \mathrm{M})$ to prevent the feedback activation of PDE4. The PKA inhibitor itself did not produce a change in basal cAMP activity (Fig. 4); however, H89 did appear to alter the Iso-stimulated cAMP 


\section{Epac2-MyrPalm}

CONTROL
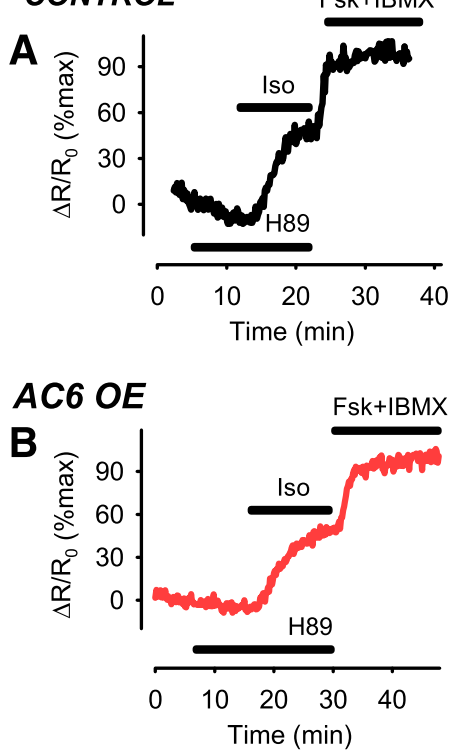

C

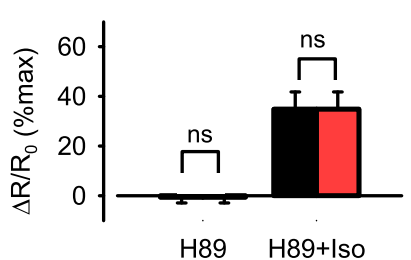

Epac2-CAAX
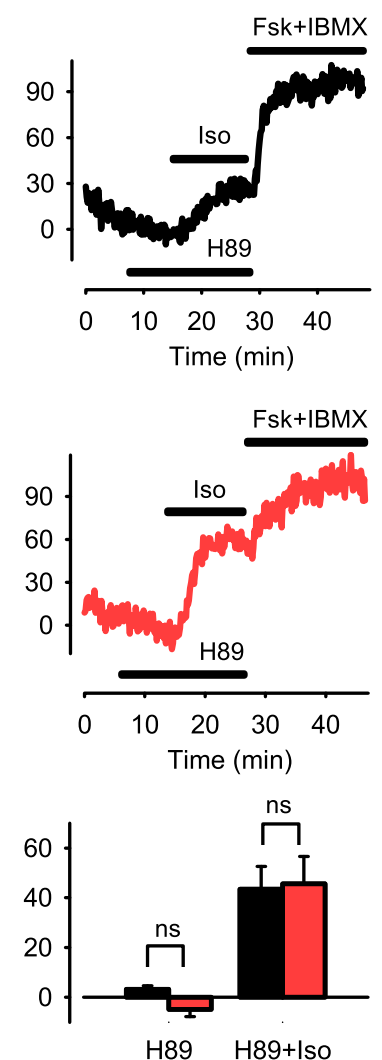

Fig. 4. Changes in cAMP activity associated with lipid raft and nonraft plasma membrane domains after $\beta_{2} \mathrm{AR}$ stimulation in the presence of $\mathrm{PKA}$ inhibition. Representative time course of changes in magnitude of FRET response $\left(\Delta \mathrm{R} / \mathrm{R}_{0}\right)$ detected by Epac2-MyrPalm and Epac2-CAAX biosensors after inhibition of PKA activity with H89 $(1 \mu \mathrm{M})$ and subsequent activation of $\beta_{2} \mathrm{ARs}$ with $3 \mathrm{nM}$ isoproterenol (Iso) in (A) untreated human ASM cells (control) and (B) human ASM cells overexpressing (OE) AC6. Results are normalized to the magnitude of the maximal response produced by forskolin (FSK, $10 \mu \mathrm{M})$ and IBMX $(100 \mu \mathrm{M})$ in the same cell. (C) Size of average FRET responses in control cells (black bars) and AC6 OE cells (red bars). In the presence of H89, there were no significant $(P>0.05$, Student's $t$ test) differences in the magnitude of the responses to Iso detected by Epac2-MyrPalm in control $(\mathrm{n} / N=6 / 4)$ and AC6 OE $(\mathrm{n} / N=6 / 4)$ cells or those detected by Epac2-CAAX in control $(\mathrm{n} / N=9 / 7)$ and AC6 OE $(\mathrm{n} / N=6 / 4)$ cells.

response detected by Epac2-MyrPalm in AC6 overexpressing cells (Fig. 4B). In the presence of $\mathrm{H} 89$, the response to $3 \mathrm{nM}$ Iso was not significantly different from control cells $(P>0.05)$ (Fig. 4C). Again, this is in contrast to the significant decrease in magnitude of the $\beta_{2} \mathrm{AR}$ response associated with AC6 overexpression in the absence of H89 (see Fig. 1, B and C). This effect appeared to be specific for responses associated with lipid raft domains as H89 did not enhance the $\beta_{2} \mathrm{AR}$ responses detected in nonraft domains of AC6- overexpressing cells (Fig. 4C).

The preceding results support the idea that positive feedback activation of PDE4 contributes to the decrease in cAMP produced in response to $\beta_{2} \mathrm{AR}$ stimulation in lipid raft domains of the plasma membrane, but only in AC6 overexpressing cells. This could be explained if overexpression of AC6 leads to recruitment of excess PKA activity. Adenylyl cyclases are integral components of signaling complexes associated with type II PKA through interactions with A kinase anchoring proteins (AKAPs) (Dessauer, 2009). To

Epac2-MyrPalm

Epac2-CAAX
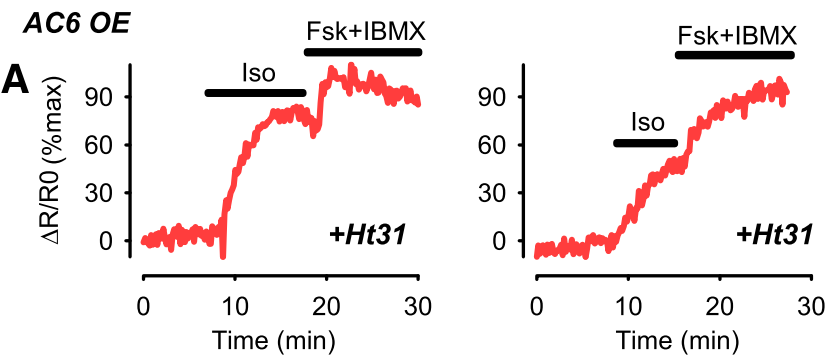

$\mathbf{B}$
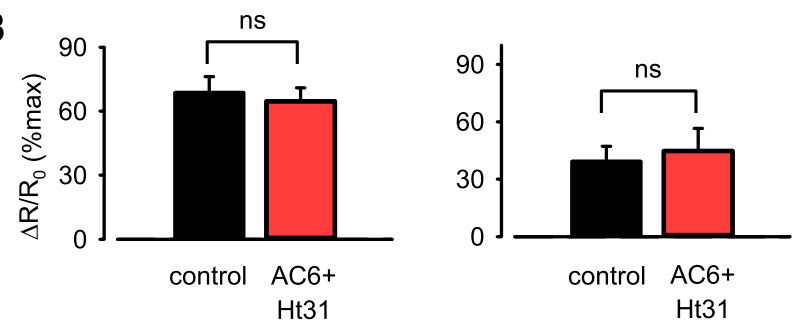

Fig. 5. Changes in cAMP activity associated with lipid raft and nonraft plasma membrane domains after $\beta_{2} \mathrm{AR}$ stimulation in cells overexpressing the AKAP-disrupting peptide Ht31. (A) Representative time course of changes in magnitude of FRET response $\left(\Delta R / R_{0}\right)$ detected by Epac2MyrPalm and Epac2-CAAX biosensors after activation of $\beta_{2} \mathrm{ARs}$ with $3 \mathrm{nM}$ isoproterenol (Iso) in human ASM cells overexpressing (OE) Ht31 and AC6 $\mathrm{OE}$. Results are normalized to the magnitude of the maximal response produced by forskolin (FSK, $10 \mu \mathrm{M})$ and IBMX $(100 \mu \mathrm{M})$ in the same cell. (B) Size of average FRET responses in control cells (black bars) and cells overexpressing AC6 plus Ht31 (red bars). There were no significant $(P>0.05$, Student's $t$ test with Holm-Sidak correction) differences in the magnitude of the responses to Iso detected by Epac2-MyrPalm in control cells $(\mathrm{n} / N=6 / 6)$ and cells overexpressing AC6 plus Ht31) $(\mathrm{n} / N=10 / 4)$ or the magnitude of the responses detected by Epac2-CAAX in control cells $(\mathrm{n} / N=7 / 6)$ and cells overexpressing $\mathrm{Ht} 31$ plus AC6 $(\mathrm{n} / N=7 / 3)$. Control data from experiments illustrated in Fig. 1.

determine whether this type of interaction contributes to the effect of AC6 overexpression, we compared responses to Iso in cells overexpressing the Ht31 peptide, which disrupts PKA anchoring to AKAPs (Carr et al., 1992). We found that in the presence of the Ht31 peptide, the response to $3 \mathrm{nM}$ Iso detected by Epac2-MyrPalm in cells overexpressing AC6 was no different in magnitude from the response observed in control cells $(P>0.05)$ (Fig. 5). This finding suggests that the decrease in magnitude of the Iso response detected by Epac2MyrPalm in AC6-overexpressing cells in the absence of Ht31 (see Fig. 1, B and C) involves the recruitment of PKA activity to lipid raft domains where $\beta_{2} \mathrm{AR}$ signaling occurs. Again, this effect appears to be specific for responses associated with lipid raft domains as Ht31 did not alter the Iso response detected by Epac2-CAAX $(P>0.05)$ (Fig. 5).

\section{Discussion}

In the present study, we found that activation of $\beta_{2} \mathrm{ARs}$ with Iso caused an increase in cAMP activity that could be detected throughout the cytoplasmic domain of human ASM cells, as well as subcellular locations associated with lipid raft and nonraft domains of the plasma membrane; however, changes associated with lipid raft domains were more sensitive to $\beta_{2} \mathrm{AR}$ stimulation and larger than those associated with nonraft domains. Furthermore, overexpression of AC6 selectively altered $\beta_{2} \mathrm{AR}$ production of cAMP in lipid raft domains, without affecting cAMP responses occurring in other locations 
non-raft domain

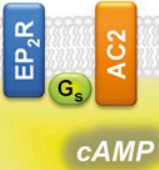

lipid raft domain

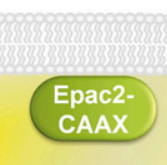

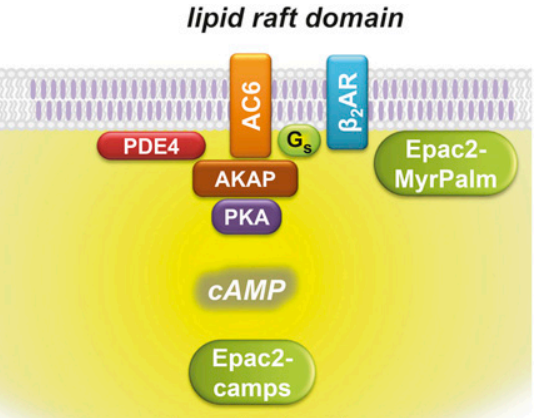

cytosolic domain
Fig. 6. Compartmentalized cAMP signaling in human airway smooth muscle cells. $\beta_{2} \mathrm{AR}$ stimulation of AC6 produces cAMP that is most readily detected by the Epac2MyrPalm biosensor, which is targeted to lipid raft domains of the plasma membrane, and the Epac2-camps biosensor, which is expressed throughout the cytosolic domain. AKAPs contribute to the formation of a signaling complex that includes $\beta_{2} \mathrm{ARs}$, AC6, type II PKA, and PDE4 associated with lipid raft membrane domains. $\mathrm{EP}_{2} \mathrm{Rs}$ stimulation of $\mathrm{AC}$ type 2 (AC2) produces cAMP that is most readily detected by the Epac2-CAAX biosensor, which is targeted to nonraft membrane domains. or cAMP responses mediated by other receptors. In addition, the effect that AC6 overexpression had on $\beta_{2} \mathrm{AR}$ responses in lipid raft domains was inhibitory, and this inhibitory effect could be blocked by inhibition of PDE4 activity, PKA kinase activity, as well as AKAP interactions with PKA.

The ability of AC6 overexpression to selectively alter $\beta_{2} \mathrm{AR}$ responses associated with lipid raft domains is consistent with previous findings demonstrating that both signaling proteins are concentrated in caveolar fractions of the plasma membrane in ASMs (Bogard et al., 2011, 2012); however, the fact that the AC6 overexpression produced an inhibitory effect in the present study was somewhat surprising. It was previously reported that overexpression of AC6 enhances cAMP production in response to direct activation of $\mathrm{AC}$ activity with forskolin or $\beta_{2} \mathrm{ARs}$ with Iso. This apparent contradiction is most likely explained by the fact that the earlier studies measured cAMP activity in cell lysates using traditional immunoassay techniques that required the use of maximally stimulating concentrations of the nonspecific PDE inhibitor IBMX. The results of the present study suggest that the effects of AC6 overexpression are more subtle in that they appear to occur only in specific subcellular locations and can be blocked by selective inhibition of PDE4 activity. Therefore, the use of whole-cell lysates and IBMX would have obscured the ability to detect the kind of response found presently using targeted biosensors in intact cells.

The ability to block the effect of AC6 overexpression on $\beta_{2} \mathrm{AR}$ responses with rolipram as well as the PKA kinase inhibitor H89 supports the idea that the inhibitory effect was due to an increase in PDE4 activity. It is well documented that PDE4 activity can be stimulated after phosphorylation by PKA (Conti and Beavo, 2007). It is conceivable that the increased expression of AC activity contributed to an initial increase in cAMP production upon $\beta_{2} \mathrm{AR}$ stimulation that resulted in a rapid activation of PKA and subsequent phosphorylation of PDE4. If this was the case, one might have expected to see a rapid increase, followed by a decrease in cAMP after exposure to Iso. One possibility is that it occurred too rapidly for us to detect, especially since we were only able to measure FRET responses once every 10 seconds. It could also be that the increase in cAMP needed to activate PKA occurred locally, within a signaling complex that may not have been accessible to the Epac2-MyrPalm biosensor, even though they are all associated with the same membrane domain.

Consistent with the idea that the $\beta_{2} \mathrm{AR}$ and AC6 are part of a signaling complex that includes PKA is the fact that the inhibitory effect of AC6 overexpression was also blocked by the AKAP inhibitory peptide Ht31. This peptide mimics the AKAP-anchoring sequence that binds to the regulatory subunit of type II PKA. Preventing the anchoring of PKA would have limited the ability of local activation of PKA to phosphorylate PDE4. The implication is that the PDE4 is also part of this signaling complex. Although we do not know which specific AKAP is involved, several different isoforms have been found to interact with certain AC subtypes and can regulate compartmentalized cAMP signaling (Horvat et al., 2012). For example, AKAP79/AKAP150/AKAP5 is able to interact with $\mathrm{AC2}, 3,5,6,8$, and 9 (Efendiev et al., 2010); yotiao/AKAP9 interacts with AC1, 2, 3, and 9 (Piggott et al., 2008 ); and mAKAP $\beta / A K A P 6$ can interact with AC2 and 5 (Kapiloff et al., 2009). Although the evidence presented in the current study supports the idea that AKAP anchoring of PKA is necessary to see the effect of AC6 overexpression, it is possible that the signaling complex, which appears to include $\beta_{2} \mathrm{ARs}$, AC6, PDE4, and PKA (Fig. 6), may involve other scaffolding proteins, such as caveolins (Ostrom et al., 2012). In either case, inhibition of PDE4 activity might have been expected to affect $\beta_{2} \mathrm{AR}$ responses detected by Epac2MyrPalm in non-AC6-overexpressing cells (see Fig. 3A). One possible reason it did not is that other PDE isoforms are present that can compensate for the loss of PDE4 activity. Only when overexpression of AC6 recruits excess PDE4 activity to that microdomain is an effect apparent.

In conclusion, using targeted FRET-based biosensors, we demonstrated that $\beta_{2} \mathrm{AR}$ stimulation produces a compartmentalized cAMP response associated with lipid raft domains of the plasma membrane in human ASM cells. Furthermore, overexpression of AC6 activity revealed functional evidence that the $\beta_{2} \mathrm{AR}$ is associated with a signaling complex that includes AC6, PKA, and PDE4. These findings imply that therapeutic strategies involving combined $\beta_{2} \mathrm{AR}$ activation and PDE4 inhibition might be particularly effective at increasing cAMP levels and bronchodilation in human ASM.

\section{Acknowledgments}

We thank Dr. Raymond Penn and Dr. Tonio Pera, Thomas Jefferson University, for supplying the human airway smooth muscle cells.

\section{Authorship Contributions}

Participated in research design: Ostrom, Harvey.

Conducted experiments: Agarwal, Fiore, Miyashiro.

Contributed new reagents or analytic tools: Harvey, Agarwal.

Performed analysis: Agarwal, Fiore, Miyashiro.

Wrote or contributed to the writing of the manuscript: Agarwal, Ostrom, Harvey. 


\section{References}

Agarwal SR, Gratwohl J, Cozad M, Yang PC, Clancy CE, and Harvey RD (2018) Compartmentalized cAMP signaling associated with lipid raft and non-raft membrane domains in adult ventricular myocytes. Front Pharmacol 9:332.

Agarwal SR, Miyashiro K, Latt H, Ostrom RS, and Harvey RD (2017) Compartmentalized cAMP responses to prostaglandin $\mathrm{EP}_{2}$ receptor activation in human airway smooth muscle cells. Br J Pharmacol 174:2784-2796.

Agarwal SR, Yang PC, Rice M, Singer CA, Nikolaev VO, Lohse MJ, Clancy CE, and Harvey RD (2014) Role of membrane microdomains in compartmentation of cAMP signaling. PLoS One 9:e95835.

Billington CK, Ojo OO, Penn RB, and Ito S (2013) cAMP regulation of airway smooth muscle function. Pulm Pharmacol Ther 26:112-120.

Bogard AS, Adris P, and Ostrom RS (2012) Adenylyl cyclase 2 selectively couples to E prostanoid type 2 receptors, whereas adenylyl cyclase 3 is not receptor-regulated in airway smooth muscle. J Pharmacol Exp Ther 342:586-595.

Bogard AS, Birg AV, and Ostrom RS (2014) Non-raft adenylyl cyclase 2 defines a cAMP signaling compartment that selectively regulates IL-6 expression in airway smooth muscle cells: differential regulation of gene expression by AC isoforms. Naunyn Schmiedebergs Arch Pharmacol 387:329-339.

Bogard AS, Xu C, and Ostrom RS (2011) Human bronchial smooth muscle cells express adenylyl cyclase isoforms 2,4 , and 6 in distinct membrane microdomains. $J$ Pharmacol Exp Ther 337:209-217.

Carr DW, Hausken ZE, Fraser ID, Stofko-Hahn RE, and Scott JD (1992) Association of the type II cAMP-dependent protein kinase with a human thyroid RII-anchoring protein. Cloning and characterization of the RII-binding domain. J Biol Chem $\mathbf{2 6 7}$ 13376-13382.

Conti M and Beavo J (2007) Biochemistry and physiology of cyclic nucleotide phosphodiesterases: essential components in cyclic nucleotide signaling. Annu Rev Biochem 76:481-511.

Depry C, Allen MD, and Zhang J (2011) Visualization of PKA activity in plasma membrane microdomains. Mol Biosyst 7:52-58.

Dessauer CW (2009) Adenylyl cyclase-A-kinase anchoring protein complexes: the next dimension in cAMP signaling. Mol Pharmacol 76:935-941.

Efendiev R, Samelson BK, Nguyen BT, Phatarpekar PV, Baameur F, Scott JD, and Dessauer CW (2010) AKAP79 interacts with multiple adenylyl cyclase (AC) isoforms and scaffolds AC5 and -6 to alpha-amino-3-hydroxyl-5-methyl-4-isoxazolepropionate (AMPA) receptors. J Biol Chem 285:14450-14458.

Horvat SJ, Deshpande DA, Yan H, Panettieri RA, Codina J, DuBose TD Jr, Xin W, Rich TC, and Penn RB (2012) A-kinase anchoring proteins regulate compartmentalized cAMP signaling in airway smooth muscle. FASEB $J \mathbf{2 6}$ 3670-3679.

Iancu RV, Ramamurthy G, Warrier S, Nikolaev VO, Lohse MJ, Jones SW, and Harvey RD (2008) Cytoplasmic cAMP concentrations in intact cardiac myocytes. Am J Physiol Cell Physiol 295:C414-C422.

Insel PA, Head BP, Ostrom RS, Patel HH, Swaney JS, Tang CM, and Roth DM (2005) Caveolae and lipid rafts: $\mathrm{G}$ protein-coupled receptor signaling microdomains in cardiac myocytes. Ann N Y Acad Sci 1047:166-172.

Kapiloff MS, Piggott LA, Sadana R, Li J, Heredia LA, Henson E, Efendiev R, and Dessauer CW (2009) An adenylyl cyclase-mAKAPbeta signaling complex regulates cAMP levels in cardiac myocytes. $J$ Biol Chem 284: $23540-23546$.
Méhats C, Jin SLCL, Wahlstrom J, Law E, Umetsu DT, and Conti M (2003) PDE4D plays a critical role in the control of airway smooth muscle contraction. FASEB J 17:1831-1841.

Melkonian KA, Ostermeyer AG, Chen JZ, Roth MG, and Brown DA (1999) Role of lipid modifications in targeting proteins to detergent-resistant membrane rafts. Many raft proteins are acylated, while few are prenylated. J Biol Chem 274:3910-3917.

Nikolaev VO, Bünemann M, Hein L, Hannawacker A, and Lohse MJ (2004) Novel single chain cAMP sensors for receptor-induced signal propagation. J Biol Chem 279:37215-37218.

Noble PB, Pascoe CD, Lan B, Ito S, Kistemaker LE, Tatler AL, Pera T, Brook BS Gosens R, and West AR (2014) Airway smooth muscle in asthma: linking contraction and mechanotransduction to disease pathogenesis and remodelling. Pulm Pharmacol Ther 29:96-107.

Ostrom RS, Bogard AS, Gros R, and Feldman RD (2012) Choreographing the adenylyl cyclase signalosome: sorting out the partners and the steps. Naunyn Schmiedebergs Arch Pharmacol 385:5-12.

Ostrom RS, Gregorian C, Drenan RM, Xiang Y, Regan JW, and Insel PA (2001) Receptor number and caveolar co-localization determine receptor coupling efficiency to adenylyl cyclase. J Biol Chem 276:42063-42069.

Ostrom RS and Insel PA (2006) Methods for the study of signaling molecules in membrane lipid rafts and caveolae. Methods Mol Biol 332:181-191.

Pelaia G, Renda T, Gallelli L, Vatrella A, Busceti MT, Agati S, Caputi M, Cazzola M, Maselli R, and Marsico SA (2008) Molecular mechanisms underlying airway smooth muscle contraction and proliferation: implications for asthma. Respir Med 102:1173-1181.

Penn RB, Panettieri RA Jr, and Benovic JL (1998) Mechanisms of acute desensitization of the beta2AR-adenylyl cyclase pathway in human airway smooth muscle. Am J Respir Cell Mol Biol 19:338-348.

Piggott LA, Bauman AL, Scott JD, and Dessauer CW (2008) The A-kinase anchoring protein Yotiao binds and regulates adenylyl cyclase in brain. Proc Natl Acad Sci USA 105:13835-13840.

Taylor DR (2009) The beta-agonist saga and its clinical relevance: on and on it goes. Am J Respir Crit Care Med 179:976-978.

Warrier S, Belevych AE, Ruse M, Eckert RL, Zaccolo M, Pozzan T, and Harvey RD (2005) Beta-adrenergic- and muscarinic receptor-induced changes in cAMP activity in adult cardiac myocytes detected with FRET-based biosensor. Am J Physiol Cell Physiol 289:C455-C461.

Warrier S, Ramamurthy G, Eckert RL, Nikolaev VO, Lohse MJ, and Harvey RD (2007) cAMP microdomains and L-type $\mathrm{Ca} 2+$ channel regulation in Guinea-pig ventricular myocytes. J Physiol 580:765-776.

Yan H, Deshpande DA, Misior AM, Miles MC, Saxena H, Riemer EC, Pascual RM, Panettieri RA, and Penn RB (2011) Anti-mitogenic effects of $\beta$-agonists and PGE2 on airway smooth muscle are PKA dependent. FASEB J 25:389-397.

Zacharias DA, Violin JD, Newton AC, and Tsien RY (2002) Partitioning of lipidmodified monomeric GFPs into membrane microdomains of live cells. Science $\mathbf{2 9 6}$ : 913-916.

Address correspondence to: Robert D. Harvey, Department of Pharmacology, University of Nevada, Reno, 1664 N. Virginia Street, MS 573, Reno, NV 89557. E-mail: rdharvey@med.unr.edu 